\title{
Synthesis of $\mathrm{Ca}_{3} \mathrm{Co}_{4} \mathrm{O}_{9}$ Ceramics by Citric Acid Complex and Hydrothermal Hot-Pressing Processes and Investigation of Its Thermoelectric Properties
}

\author{
Shigeru Katsuyama, Yuta Takiguchi* and Mikio Ito \\ Division of Materials and Manufacturing Science, Graduate School of Engineering, Osaka University, Suita 565-0871, Japan
}

$\mathrm{Ca}_{3} \mathrm{Co}_{4} \mathrm{O}_{9}$ ceramics powders were synthesized by the citric acid complex (CAC) method and consolidated by the hydrothermal hotpressing (HHP) technique. The observation by the scanning electron microscope indicated that the powders obtained by the CAC process showed plate-like grains. The density and the Lotgering factor, which was estimated from the X-ray diffraction data, of the sintered body increased with an increase of the operating pressure during the HHP process. The electrical resistivity was much reduced with an increase of the operating pressure, but the Seebeck coefficient was hardly affected by the HHP conditions. As a result, the sample treated with the HHP under the condition of $573 \mathrm{~K}, 200 \mathrm{MPa}$ and $3 \mathrm{~h}$ showed a maximum power factor. [doi:10.2320/matertrans.E-MRA2007805]

(Received November 14, 2006; Accepted February 4, 2007; Published June 6, 2007)

Keywords: citric acid complex, hydrothermal hot-pressing, Seebeck coefficient, electrical resistivity, Lotgering factor

\section{Introduction}

Recently metal oxides have attracted much attention of many researchers as high temperature thermoelectric materials because of their high stability to heat and low toxicity. $\mathrm{Ca}_{3} \mathrm{Co}_{4} \mathrm{O}_{9}$ is a layered oxide composed of an electrically conductive $\mathrm{CoO}_{2}$ layer and an insulating $\mathrm{Ca}_{2} \mathrm{CoO}_{3}$ layer which are aligned along the $c$-axis. The electrical resistivity of this phase exhibits significant anisotropy due to its crystal structure; the electrical resistivity along the $c$-axis is considerably higher than that along the $c$-plane. ${ }^{1-3)}$ And so, for the achievement of high $Z T$, the synthesis of the $\mathrm{Ca}_{3} \mathrm{Co}_{4} \mathrm{O}_{9}$ polycrystalline sintered body with high crystallographic orientation has been desired.

The $\mathrm{Ca}_{3} \mathrm{Co}_{4} \mathrm{O}_{9}$ sintered body is generally synthesized from a mixture of $\mathrm{CaCO}_{3}$ and $\mathrm{Co}_{3} \mathrm{O}_{4}$ powders through the calcination and subsequent sintering. It is difficult to synthesize a sintered body with high crystallographic orientation and fine microstructure by such a conventional solid-state reaction method. The citric acid complex method (CAC), which is a kind of chemical solution process, is considered to be useful for obtaining a homogeneous and fine flaky powder precursor. This method is based on the stable formation of metal-citrate complexes and the following heating in air causes a breakdown of the complexes, which allows the fabrication of the homogeneous metal oxide precursors. $\mathrm{Na}_{\mathrm{x}} \mathrm{Co}_{2} \mathrm{O}_{4}$ is also known as a material with high crystallographic anisotropy; the electrical resistivity along its $c$-axis is about 10 times larger than that along its $a$-axis. ${ }^{4)}$ One of the authors reported that a flaky powder precursor of $\mathrm{Na}_{\mathrm{x}} \mathrm{Co}_{2} \mathrm{O}_{4}$ with crystallographic anisotropy reflecting its layered structure was obtained by the CAC method and the following sintering of the powder precursor resulted in the polycrystalline sintered body with the $c$-axis of each grain aligned along the pressing direction.5) In this previous investigation, the calcined powder precursor was subjected to the pressure-less sintering in air. In the case of the pressure-less sintering, the heat treatment at high temperature and for a long time is necessary for obtaining the dense

*Graduate Student, Osaka University sintered materials.

On the other hand, the hydrothermal hot-pressing technique (HHP) is a method by which hard solid bodies can be produced from powders in a short time and at a relatively low temperature under the saturated vapor pressure. ${ }^{6,7)}$ The following sintering of the solid bodies prepared by the HHP may produce the dense sintered body resulting in the low electrical resistivity. In fact, we previously succeeded in obtaining the fully dense sintered $\mathrm{Na}_{\mathrm{x}} \mathrm{Co}_{2} \mathrm{O}_{4}$ ceramics with the plate-like grains aligned along the pressed plane by the combination of the CAC and HHP methods. ${ }^{8)}$

In this study, we have tried to prepare $\mathrm{Ca}_{3} \mathrm{Co}_{4} \mathrm{O}_{9}$ ceramics by the CAC method and HHP technique, and examined their microscopic structure and thermoelectric properties.

\section{Experimental Procedure}

$\mathrm{Ca}_{3} \mathrm{Co}_{4} \mathrm{O}_{9}$ ceramics were synthesized by the $\mathrm{CAC}$ method and HHP technique as summarized in Fig. 1. Citric acid was first dissolved in water, and subsequently $\left(\mathrm{CH}_{3}\right.$ $\mathrm{COO})_{2} \mathrm{Ca} \cdot \mathrm{H}_{2} \mathrm{O}$ and $\left(\mathrm{CH}_{3} \mathrm{COO}\right)_{2} \mathrm{Co} \cdot 4 \mathrm{H}_{2} \mathrm{O}$ in a molar ratio corresponding to the composition of $\mathrm{Ca}_{3} \mathrm{Co}_{4} \mathrm{O}_{9}$ were added to this solution. The citric acid was mixed in the proportion of 4 moles for each mole of metal cation. The solution was stirred and gradually heated, and condensed at $433 \mathrm{~K}$. During this heating process, the formation of the metal citrate complexes was prompted. As the solution was condensed, it became viscous, and this viscous product was decomposed to a fine powder at $723 \mathrm{~K}$. The obtained powder precursor was ground and calcined at $973 \mathrm{~K}$ for $12 \mathrm{~h}$ in air to remove a residual organic matter completely. The calcined powder precursor was kneaded with saturated water with $\mathrm{Ca}(\mathrm{OH})_{2}$ (10 mass\% for the powder) to be set in the cylinder of an autoclave for the hydrothermal hot-pressing. Figure 2 shows the schematic drawing of the autoclave used in this experiment. The autoclave has a cylinder made of the stainless steel whose inside diameter is 10 or $20 \mathrm{~mm}$ and the sample is compressed by the piston in the cylinder from the top and bottom. ${ }^{6)}$ The sample was first compressed at ambient temperature and the desired pressures (50-200 MPa) and then heated at a rate of $10 \mathrm{~K} \mathrm{~min}^{-1}$ to the desired temper- 


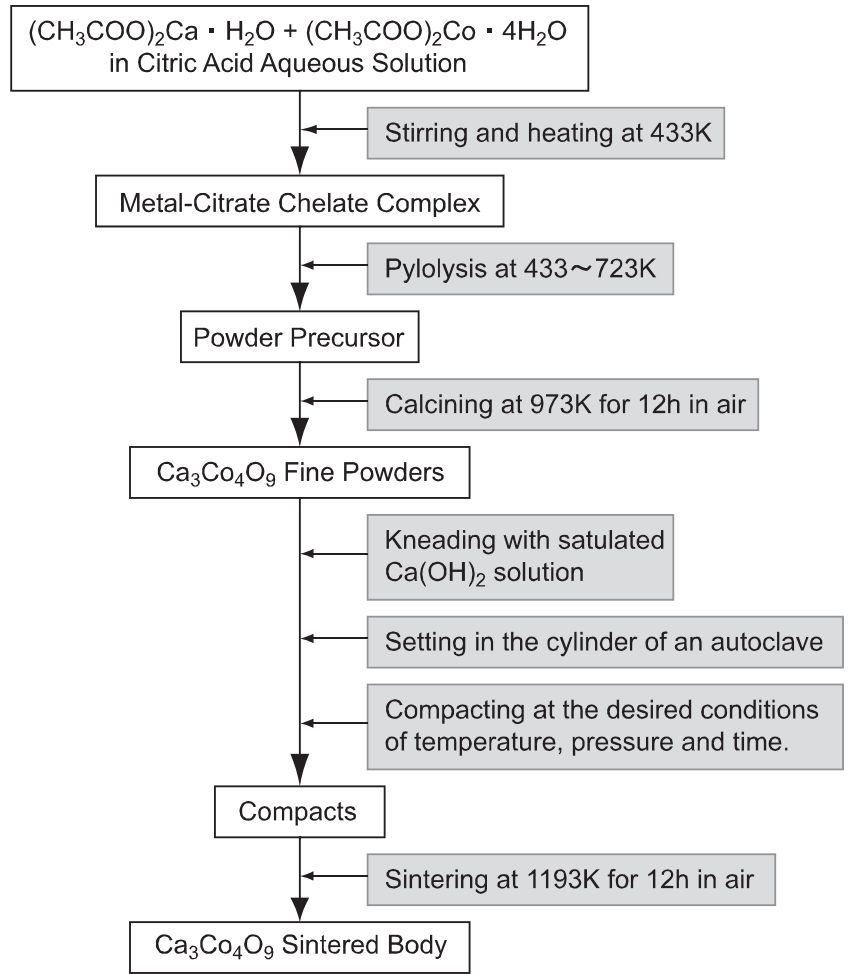

Fig. 1 Flow chart of the sample preparation.

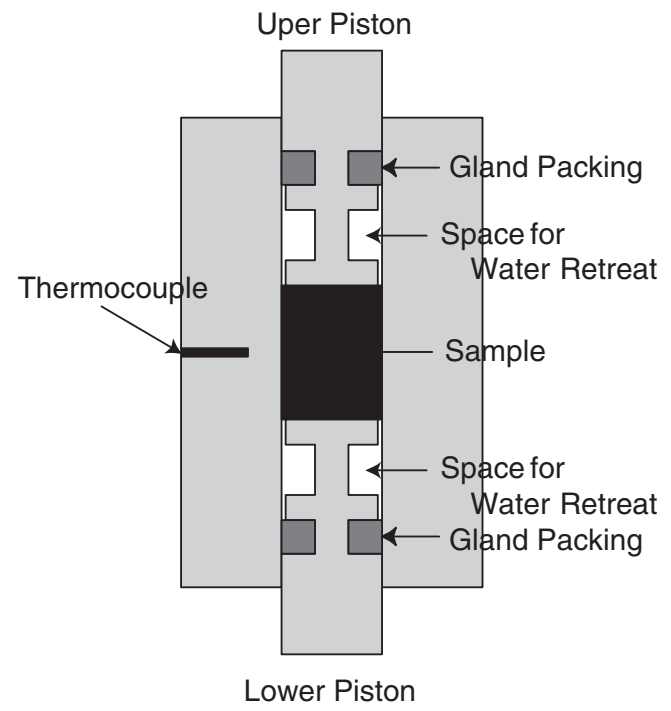

Fig. 2 Schematic drawing of the autoclave for the hydrothermal hotpressing.

atures $(473-573 \mathrm{~K})$ and kept for the desired times $(1-5 \mathrm{~h})$ at that temperature. The rectangular prism with about $18 \mathrm{~mm} \times$ $7 \mathrm{~mm} \times 2 \mathrm{~mm}$ was cut off from the obtained compacts. The cut off compacts were sintered at $1193 \mathrm{~K}$ for $12 \mathrm{~h}$ in air. As a reference, the sample which was not treated with HHP was also synthesized; the obtained powder precursor by the CAC method was pressed into a pellet under a pressure of $560 \mathrm{MPa}$ for $30 \mathrm{~s}$ at room temperature and then sintered at $1193 \mathrm{~K}$ for $12 \mathrm{~h}$ in air.

The phase identification of the obtained samples was made by X-ray diffraction at room temperature. The micro-

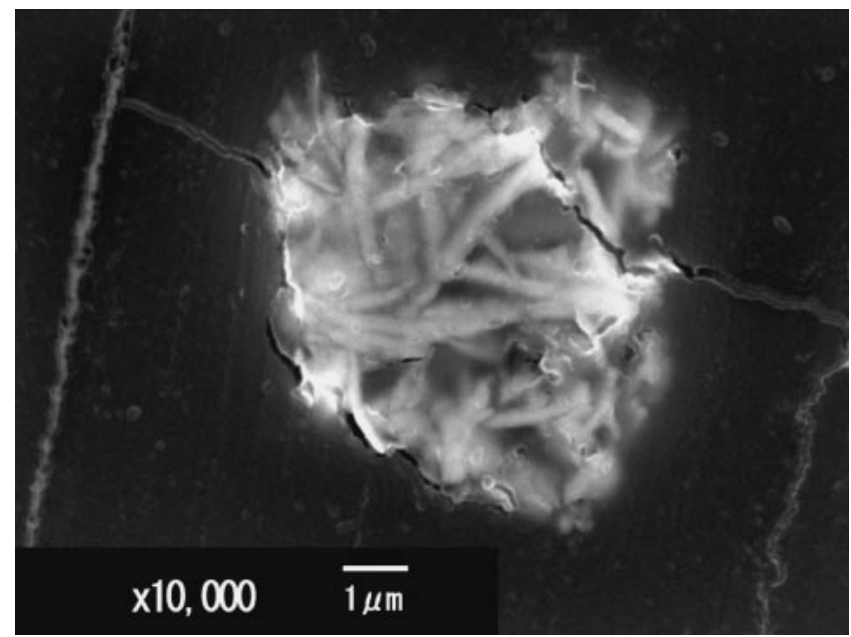

Fig. 3 SEM photograph for $\mathrm{Ca}_{3} \mathrm{Co}_{4} \mathrm{O}_{9}$ powders prepared by the citric acid complex method.

scopic structure of the samples was examined using a scanning electron microscope (SEM). The density $D$ of the samples was measured by the Archimedes method. The Seebeck coefficient $S$ and the electrical resistivity $\rho$ were simultaneously measured by the standard four-probe $\mathrm{dc}$ method in air in the temperature range from room temperature to $1173 \mathrm{~K}$ using the sintered samples shaped in the rectangular prism. The measurement was carried out along the pressed plane. The thermal diffusivity $\lambda$ and specific heat $C_{\mathrm{p}}$ were measured by the laser flash method on some samples. The measurement of $\lambda$ was carried out along the pressing direction. The thermal conductivity $\kappa$ of the samples was calculated from the measured $\lambda, C_{\mathrm{p}}$ and $D$ using $\kappa=\lambda C_{\mathrm{p}} D$.

\section{Results and Discussion}

From the result of the X-ray diffraction, it was found that the calcined powder precursor was composed of $\mathrm{Ca}_{3} \mathrm{Co}_{4} \mathrm{O}_{9}$ phase without any impurity phase. Figure 3 shows the SEM photograph for the powder precursors prepared by the CAC process. As shown in the photograph, the shape of the crystal grain is flaky, reflecting the crystal structure of $\mathrm{Ca}_{3} \mathrm{Co}_{4} \mathrm{O}_{9}$. The finally obtained sintered samples were also composed of $\mathrm{Ca}_{3} \mathrm{Co}_{4} \mathrm{O}_{9}$ phase with almost no impurity phase, and the intensity of the $(00 l)$ peaks, such as (002), (004) etc., corresponding to the $c$-plane of $\mathrm{Ca}_{3} \mathrm{Co}_{4} \mathrm{O}_{9}$ phase was relatively large compared to the other peaks. As the X-ray was irradiated to the pressed plane of the samples, this behavior seems to show that the $c$-axis direction of each grain in the sintered samples is aligned along the pressing direction (perpendicular to the pressed plane).

The degree of the crystallographic orientation of the samples can be estimated from the intensity of the $(h k l)$ peaks, $I(h k l)$, in the $\mathrm{X}$-ray diffraction pattern using the Lotgering factor, $f=\left(P-P_{0}\right) /\left(1-P_{0}\right) .{ }^{9)}$ When estimating the degree of the orientation along the $c$-axis, $P$ is defined as $\Sigma I(00 l) / \Sigma I(h k l) ; P=P_{0}$ for a crystallographically isotropic (randomly orientated) sample. In this study, $P_{0}$ was calculated from the peak data of the JCPDS card, No. 23-110. For 

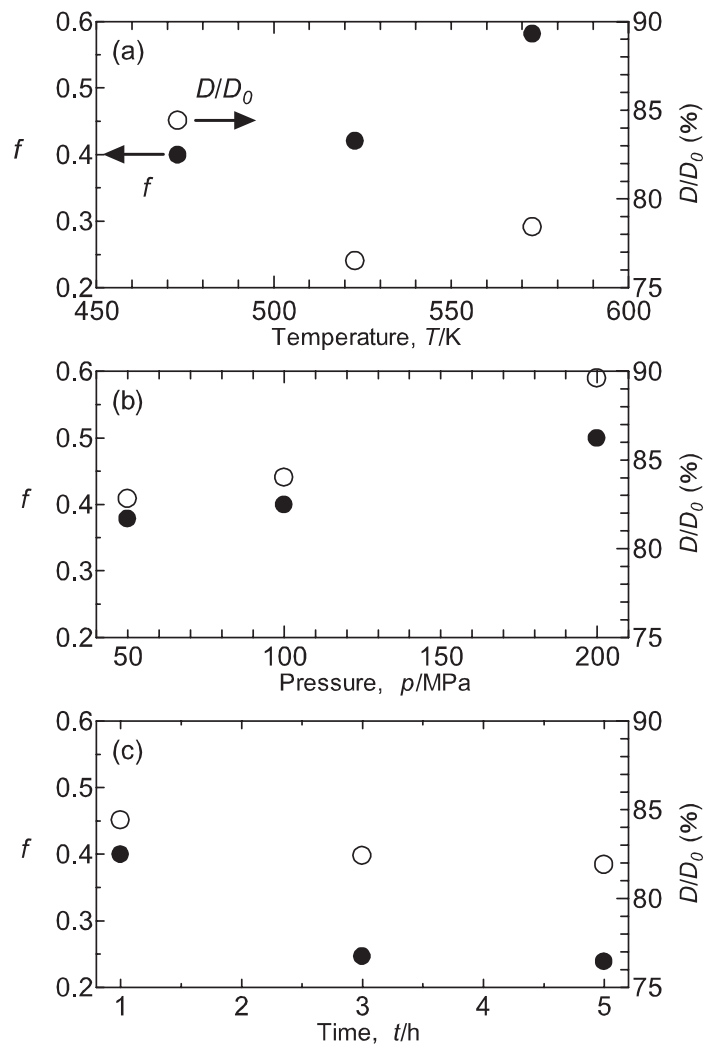

Fig. $4 f$-value and relative density $D / D_{0}$ of the samples as functions of the operating temperature, pressure and time.

the sample with the $c$-axis completely aligned along the pressing direction, the $f$-value is calculated to be 1 .

The calculated $f$-value together with the relative density $D / D_{0}$ of the sintered samples are shown in Fig. 4(a), (b) and (c) as functions of the operating temperature, pressure and time during the HHP process, respectively. Here, the operating temperature, pressure and time except the variable of the horizontal axis are fixed as $473 \mathrm{~K}, 100 \mathrm{MPa}$ and $1 \mathrm{~h}$, respectively; for example in Fig. 4(a) the operating pressure and time are fixed as $100 \mathrm{MPa}$ and $1 \mathrm{~h}$, respectively. $D$ is the density of the samples measured by the Archimedes method, while $D_{0}$ is the theoretical density calculated from the X-ray diffraction data. The $f$-value generally increases with an increase of the operating temperature and pressure, while it decreases with increasing time in the HHP process. The relative density $D / D_{0}$ of the samples tends to decrease with increasing temperature and time, but it increases with increasing pressure. These results show that the higher pressure during the HHP process is more effective in increasing both $f$-value and relative density of the samples.

We have examined the effect of the operating temperature during the HHP process on the Seebeck coefficient, electrical resistivity and power factor of the samples. Figure 5(a), (b) and (c) show the temperature dependence of the Seebeck coefficient, electrical resistivity and power factor for the samples treated by HHP under the individual operating temperatures, respectively. The operating pressure and time are fixed as $100 \mathrm{MPa}$ and $1 \mathrm{~h}$, respectively. The measurements of these properties were carried out along the pressed plane. The Seebeck coefficient gradually increases with
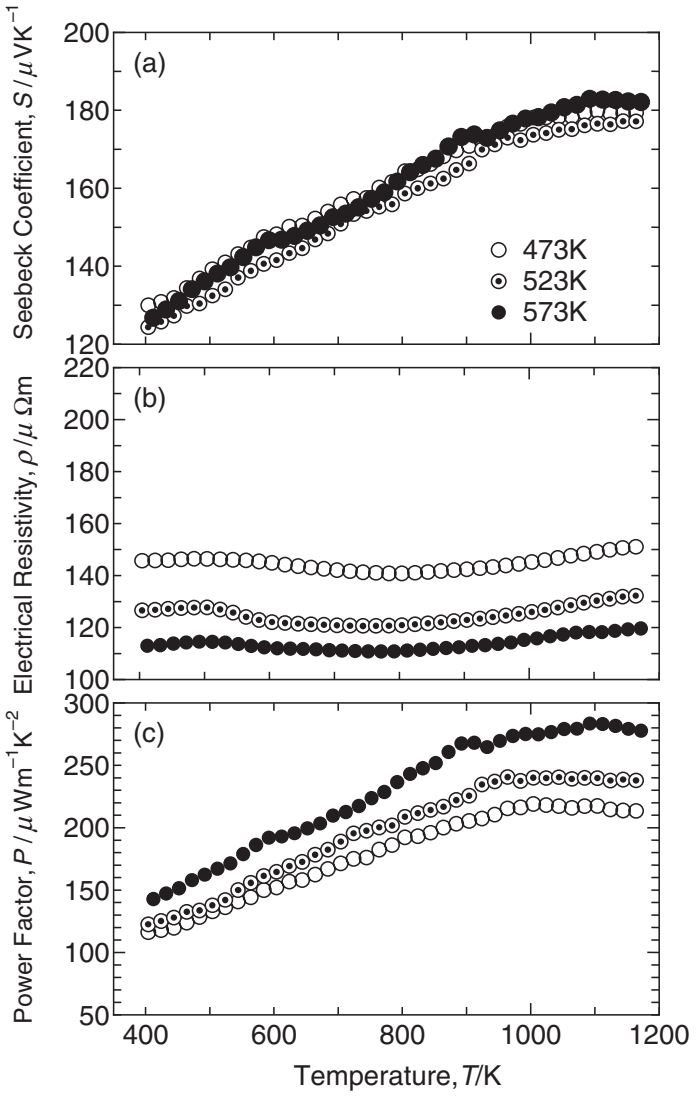

Fig. 5 Temperature dependence of the (a) Seebeck coefficient, (b) electrical resistivity and (c) power factor for the samples treated by HHP process under the individual operating temperature.

temperature, while the electrical resistivity is almost constant and independent of temperature. It is reported that the electrical resistivity in the $c$-plane of the single crystal increases with temperature while that along the $c$-axis decreases. ${ }^{3)}$ On the other hand, it is reported that the Seebeck coefficient in the $c$-plane increases with temperature, ${ }^{3)}$ and there is almost no isotropy on the Seebeck coefficient. ${ }^{10,11)}$ The temperature dependence observed in our samples is similar to the data for ceramics reported by $\mathrm{Li}$ et al. ${ }^{11)}$ The Seebeck coefficient is almost independent of the operating temperature during the HHP process, which shows that the carrier density of the samples is almost the same among these samples. The electrical resistivity generally decreases with an increase of the operating temperature during the HHP process. Considering the result on the $f$-value as shown in Fig. 4, the decrease of the electrical resistivity may be ascribed to the increase of the crystallographic orientation of the samples; the electrical resistivity of $\mathrm{Ca}_{3} \mathrm{Co}_{4} \mathrm{O}_{9}$ along the $c$-plane is smaller than that along $c$-axis. ${ }^{1-3)}$ As shown in Fig. 5(c), the power factor of the samples increases with an increase of the operating temperature during the HHP process.

Next, we have examined the effect of the operating pressure during the HHP process on the thermoelectric properties of the samples, which is shown in Fig. 6. The operating temperature and time during the HHP process are fixed as $473 \mathrm{~K}$ and $1 \mathrm{~h}$, respectively. The Seebeck coefficient is almost independent of the operating pressure, while the 


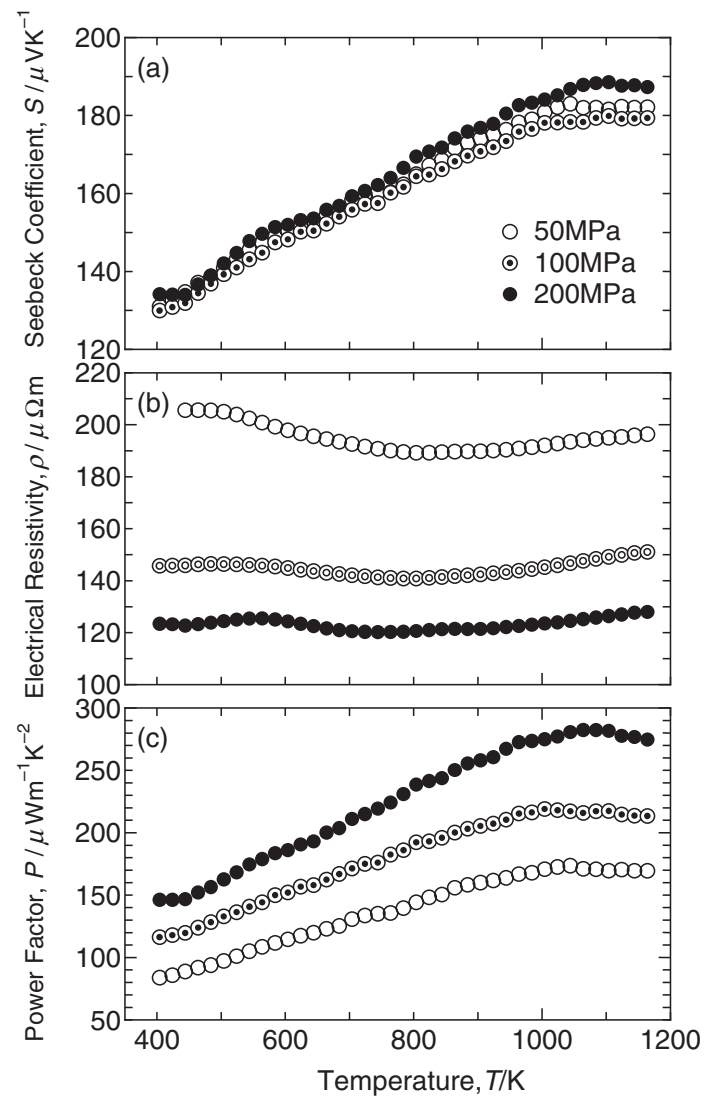

Fig. 6 Temperature dependence of the (a) Seebeck coefficient, (b) electrical resistivity and (c) power factor for the samples treated by HHP process under the individual operating pressure.

electrical resistivity is much reduced with an increase of the operating pressure. The reduction of the electrical resistivity is ascribed to the increase of the $f$-value as well as the relative density of the samples with an increase of the operating pressure during the HHP process. As a result, the power factor of the sample also increases with an increase of the operating pressure.

Finally, we have shown the operating time dependence of the Seebeck coefficient, electrical resistivity and power factor of the samples in Fig. 7. Here, the operating temperature and pressure are fixed as $473 \mathrm{~K}$ and $100 \mathrm{MPa}$, respectively. There is almost no difference in the Seebeck coefficient, electrical resistivity and power factor among these samples, i.e., the thermoelectric properties were hardly affected by the operating time during the HHP process. The power factor of the sample operated for $3 \mathrm{~h}$ is slightly larger than that of any other samples.

From the above results, we have determined that the optimum temperature, pressure and time during the HHP process are $573 \mathrm{~K}, 200 \mathrm{MPa}$ and $3 \mathrm{~h}$, respectively. Figure 8 shows the temperature dependence of the Seebeck coefficient, electrical resistivity and power factor for the sample treated with the HHP process under this optimum condition. The data of the sample, which was prepared by pressing the powder precursor by the CAC method into a pellet under a pressure of $560 \mathrm{MPa}$ for $30 \mathrm{~s}$ at room temperature followed by sintering at $1193 \mathrm{~K}$ for $12 \mathrm{~h}$ in air, were also shown. There is almost no difference in the Seebeck coefficient between

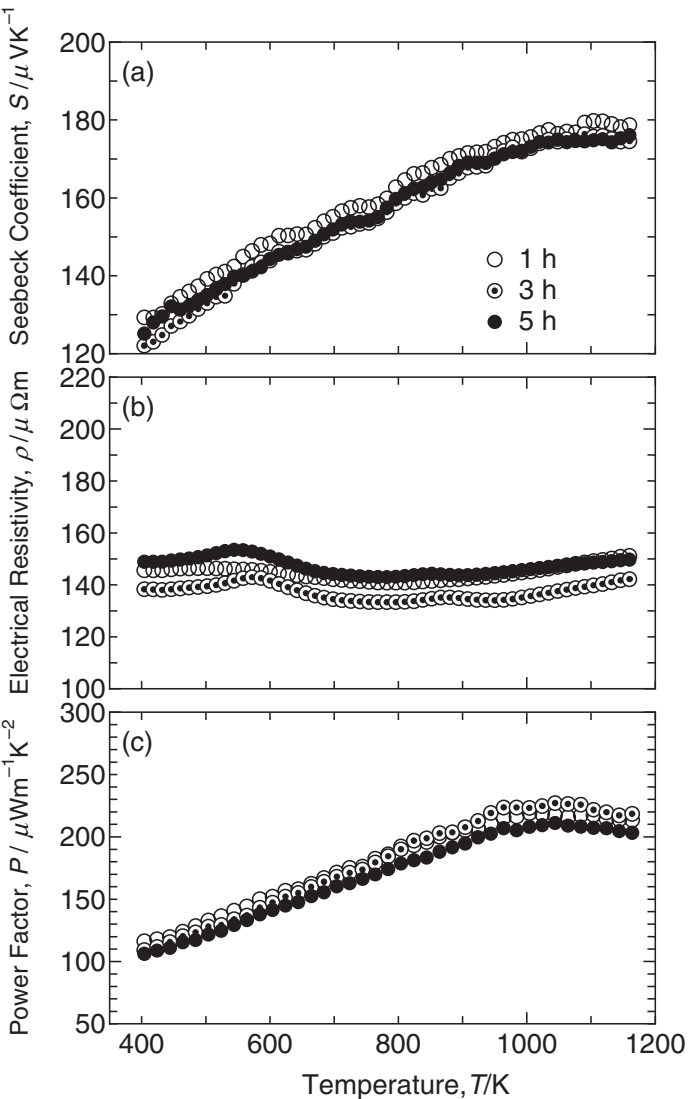

Fig. 7 Temperature dependence of the (a) Seebeck coefficient, (b) electrical resistivity and (c) power factor for the samples treated with HHP process under the individual operating time.

these two samples, but the electrical resistivity of the sample treated with the HHP process is smaller. As a result, the sample treated with the HHP process under a condition of $573 \mathrm{~K}, 200 \mathrm{MPa}$ and $3 \mathrm{~h}$ shows a maximum power factor of $315 \mu \mathrm{W} / \mathrm{m} / \mathrm{K}^{2}$ at about $1050 \mathrm{~K}$, which is larger than that of the sample untreated with the HHP process. We show the SEM photographs of the sample prepared by the HHP process under this optimum condition in Fig. 9. Here, Fig. 9(a) shows the cross-section along the pressed plane, while Fig. 9(b) shows the cross-section perpendicular to the pressed plane. As shown in the photographs, the plate-like grains are densely packed and well aligned along the pressed plane. Itahara et al. reported that the high oriented $\mathrm{Ca}_{3} \mathrm{Co}_{4} \mathrm{O}_{9}$ ceramics with $f$-value of 0.99 were obtained by the RTGG (Reactive Templated Grain Growth) method. ${ }^{12)}$ The calculated $f$-value of this our sample was 0.61 . This value is not so high when compared with the values reported by them. However, considering that $f$-value of the sample untreated by the HHP process is 0.35 , this result indicates that the $\mathrm{Ca}_{3} \mathrm{Co}_{4} \mathrm{O}_{9}$ sintered body with the plate-like grains aligned along the pressed plane can be obtained by the combination of the CAC and HHP methods.

Figure 10(a) shows the temperature dependence of the thermal conductivity for the sample treated with the HHP process under the optimum condition, together with the thermal conductivity of the sample untreated with the HHP process. The thermal conductivity of the sample treated with the HHP process is somewhat smaller than that of the sample 


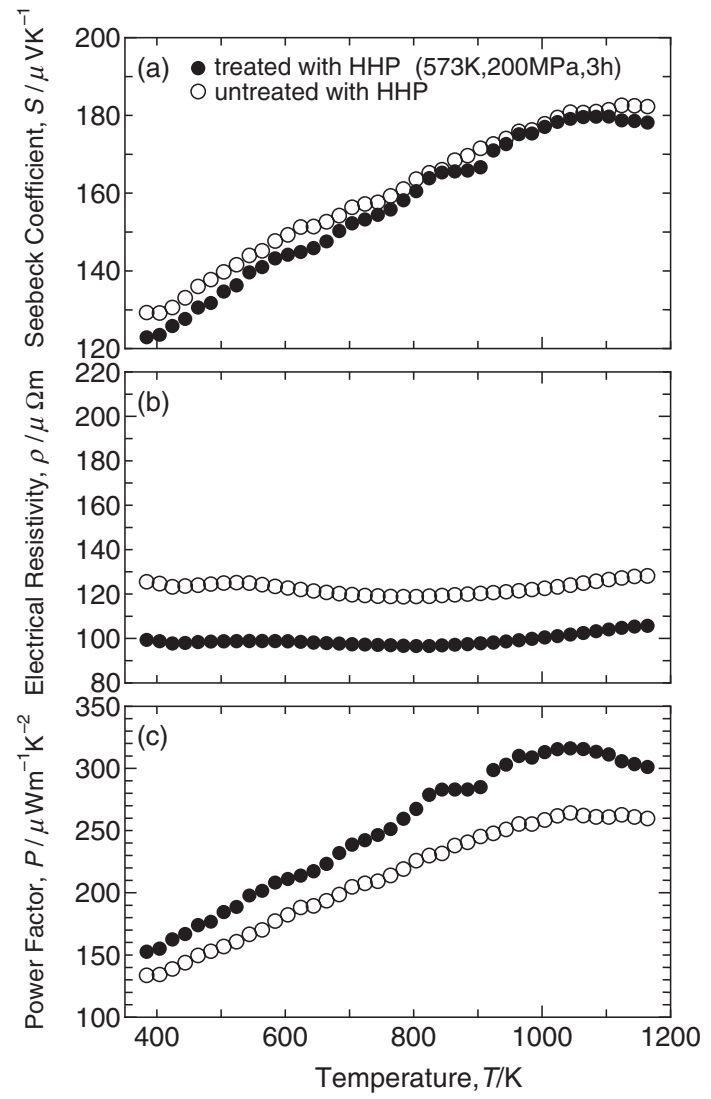

Fig. 8 Temperature dependence of the (a) Seebeck coefficient, (b) electrical resistivity and (c) power factor for the samples treated with HHP process under the optimum condition or untreated with HHP process.

untreated by the HHP process. As mentioned in the section of the experimental procedure, the measurement of the thermal diffusivity by the laser flash method was done along the pressing direction, i.e., perpendicular to the pressed plane. The somewhat decrease of the thermal conductivity in the sample treated with the HHP process seems to be ascribed to the difference of the degree of the crystallographic anisotropy between these samples; the thermal conductivity along the $c$ axis is considered to be smaller than that along the $c$-plane. ${ }^{2)}$

In order to exactly estimate the $Z T$ value of the samples with such high crystallographic anisotropy, the Seebeck coefficient, electrical resistivity and thermal conductivity must be measured along the same direction in the sintered body. As mentioned above, the measurement of the thermal diffusivity was done along the pressing direction, i.e., perpendicular to the pressed plane of the samples. On the other hand, the measurement of the Seebeck coefficient and electrical resistivity was carried out along the pressed plane of the samples. For the measurement of the thermal conductivity of the samples along the pressed plane, the sample with a thickness of more than $10 \mathrm{~mm}$ is necessary. We could not prepare such thick sintered sample at present.

Figure 10(b) shows the temperature dependence of the $Z T$, which was calculated from the measured values in the experiment, for the samples treated or untreated with the HHP process. The sample treated with the HHP under a condition of $573 \mathrm{~K}, 100 \mathrm{MPa}$ and $3 \mathrm{~h}$ shows a maximum $Z T$ of 0.28 at about $1073 \mathrm{~K}$, which is about one and a half times
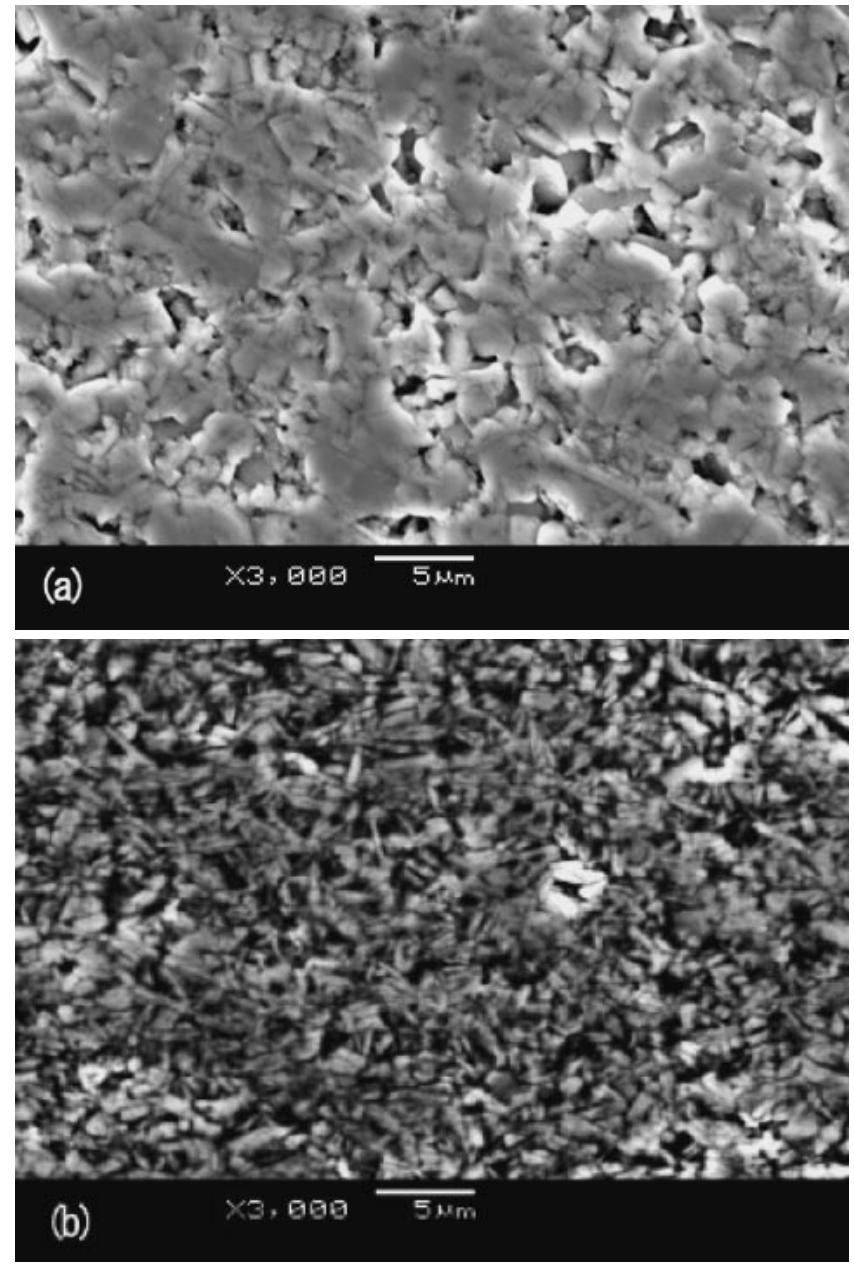

Fig. 9 SEM photographs for samples prepared by HHP process under the optimum condition. Cross-sections (a) along the pressed plane and (b) perpendicular to the pressed plane.

larger than that of the sample untreated with the HHP. This obtained maximum $Z T$ is a reference value, and in the near future, we will improve the technique of the sample preparation and estimate more accurate $Z T$ value.

\section{Conclusions}

We have prepared $\mathrm{Ca}_{3} \mathrm{Co}_{4} \mathrm{O}_{9}$ ceramics by the $\mathrm{CAC}$ method and HHP technique, and examined their microscopic structure and thermoelectric properties. The CAC method is useful for preparing a flaky powder precursor with crystallographic anisotropy reflecting its layered structure. The HHP technique is a method by which hard solid bodies can be produced from powders in a short time and at a relatively low temperature under the saturated vapor pressure. The Seebeck coefficient of the samples is hardly affected by the conditions of the HHP process, but the electrical resistivity of the samples generally decreases with an increase of the operating temperature and pressure during the HHP process. As a result, the sample treated with the HHP process under a condition of $573 \mathrm{~K}, 200 \mathrm{MPa}$ and $3 \mathrm{~h}$ shows a maximum power factor of $315 \mu \mathrm{W} / \mathrm{m} / \mathrm{K}^{2}$ at about $1050 \mathrm{~K}$. In this experiment, we could not measure the Seebeck coefficient, electrical resistivity and thermal conductivity along the same 

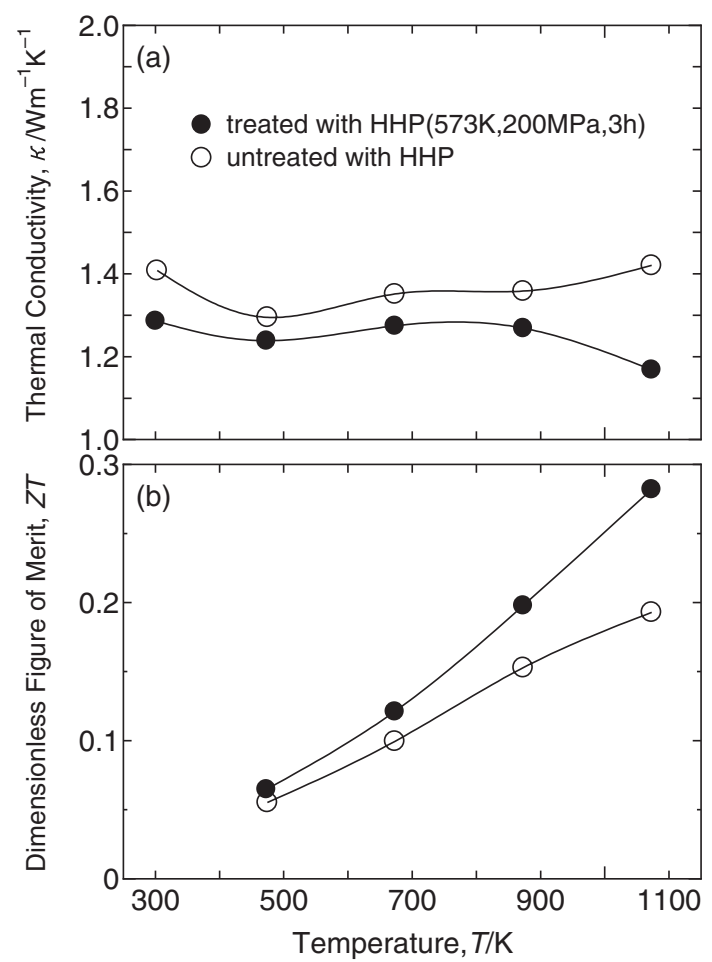

Fig. 10 Temperature dependence of the (a) thermal conductivity and (b) $Z T$ for the samples treated with HHP process under the optimum condition or untreated with HHP process.

direction in the sintered body. Although it is a reference value, a maximum $Z T$ of 0.28 at about $1073 \mathrm{~K}$ was obtained on the sample treated with the HHP under the above condition.

\section{Acknowledgement}

This study was partially supported by Industrial Technology Research Grant Program in 2004 from New Energy and Industrial Technology Development Organization (NEDO) of Japan.

\section{REFERENCES}

1) R. Funahashi, I. Matsubara, H. Ikuta, T. Takeuchi, U. Mizutani and S. Sodeoka: Jpn. J. Appl. Phys. 39 (2000) L1127-L1129.

2) J.-W. Moon, D. Nagahama, Y. Masuda, W.-S. Seo and K. Koumoto: J. Ceramic Soc. Jpn. 109 (2001) 647-650.

3) A. C. Masset, C. Michel, A. Maignam, M. Hervieu, O. Toulemonde, F. Studer and B. Raveau: Phy. Rev. B62 (2000) 166-175.

4) I. Terasaki, Y. Sasago and K. Uchinokura: Phys. Rev. B56 (1997) 12685-12687.

5) M. Ito, Y. Oda and S. Hara: J. Jpn. Soc. Powder Powder Metall. 50 (2003) 952-957.

6) N. Yamasaki, T. Kai, M. Nishioka, K. Yanagisawa and K. Ioku: J. Mater. Sci. Lett. 9 (1990) 1150-1151.

7) N. Yamasaki, T. Weiping and K. Jiajun: J. Mater. Sci. Lett. 11 (1992) 934-935.

8) S. Katsuyama, A. Kishida and M. Ito: J. Alloys and Compounds 414 (2006) 215-220.

9) F. K. Lotgering: Inorg. Nucl. Chem. 9 (1959) 113-123.

10) M. Shikame and R. Funahashi: Appl. Phys. Lett. 82 (2003) 1851-1853.

11) S. Li, R. Funahashi, I. Matsubara, K. Ueno and H. Yamada: J. Mater. Chem. 9 (1999) 1659-1660.

12) H. Itahara, C. Xia, J. Sugiyama, T. Tani and K. Koumoto: Proceedings of TEC2003, (Tokyo, 2003) pp. 172-173. 